\title{
Longer TA repeat but not V89L polymorphisms in the SRD5A2 gene may confer acne risk in the Chinese population
}

\author{
Xue Hu${ }^{1}$, Wei Ding 2 , Xinye Jin³ ${ }^{3}$ Jia Wang ${ }^{4}$, Dajin Zou', Yue Chen ${ }^{6}$ \\ 'Department of Endocrinology, Shidong Hospital, Yangpu District, Shanghai, China \\ 2Department of Geriatrics, Shanghai General Hospital, Shanghai Jiaotong University, Shanghai, China \\ ${ }^{3}$ Department of Endocrinology, Chinese People's Liberation Army General Hospital, Beijing, China \\ ${ }^{4}$ Department of Endocrinology, General Hospital of Jinan Military Region of Chinese People's Liberation Army, Jinan, China \\ ${ }^{5}$ Department of Endocrinology, Changhai Hospital Affiliated to Second Military Medical University, Shanghai, China \\ ${ }^{6}$ Department of Endocrinology, Shanghai Baoshan Traditional Chinese Medicine-integrated Hospital, Shanghai, China
}

Adv Dermatol Allergol 2018; XXXV (1): 33-38

DOI: https://doi.org/10.5114/ada.2018.73162

\begin{abstract}
Introduction: Several studies have reported that the V89L and TA repeat polymorphisms [(TA)n] of the SRD5A2 gene were associated with SRD5A2 activity. The activity of dihydrotestosterone, which is converted from testosterone by SRD5A2, is responsible for sebum secretion and the formation of acne. We hypothesized that abnormalities in SRD5A2 action could contribute to the formation of acne.

Aim: To study whether the structural change of the SRD5A2 gene may affect the risk of acne in patients with normal serum testosterone levels.

Material and methods: Genotyping of rs523349 and (TA)n of SRD5A2 was performed in 49 Chinese acne patients with significant improvements with SRD5A2 inhibitor-finasteride but normal serum testosterone levels, and in 50 healthy Chinese age-matched controls without acne.

Results: There was no significant difference between the two groups in the frequencies of $V$ and $L$ alleles and $V V$, VL, and LL genotypes of V89L ( $\chi^{2}$ test, $p>0.5$ ). (TA)n polymorphic repeat sites are 5 alleles (TA0, TA3, TA6, TA9, TA12) in our population. The differences in $S$ and $L$ allele frequencies between the two groups were statistically significant $(p<0.005)$. People with a longer $(n \geq 6)$ allele of the (TA)n repeat polymorphism had a higher risk of having acne than those with a shorter $(n<6)$ allele $(\mathrm{OR}=3.52,95 \% \mathrm{Cl}: 1.73-7.16)$.

Conclusions: This study suggests that SRD5A2 polymorphisms might be associated with acne risk. This is the first report focusing on the Chinese population according to our knowledge. Further large sample studies may be required to confirm the association and to assess any interactions with environmental factors.
\end{abstract}

Key words: acne, SRD5A2 gene, V89L polymorphism, (TA)n repeat polymorphism.

\section{Introduction}

Acne is a common chronic inflammatory disease of sebaceous glands, which are exocrine glands in the skin that secrete sebum (an oily substance) to lubricate the skin and hair. Its pathogenesis appears to be multifactorial, including hypersecretion of sebaceous glands, keratinization of the sebaceous gland ducts, and microbial infections and inflammatory mediators in pilosebaceous units [1, 2]. Androgens, as a prerequisite for sebaceous gland development and secretion of sebum, play an important role in the pathogenesis of acne. Steroid $5 \boldsymbol{\alpha}$-reductase is crucial to androgen action, which converts testosterone to the more potent androgen dihydrotestosterone (DHT). The activity of $5 \alpha$-reductase is higher in sebaceous glands of the scalp and facial skin than in sebaceous glands of nonacne-prone skin in other locations [3]. Many studies have shown that most acne patients have normal circulating androgen levels, which suggests that androgen acts on sebaceous glands independently of circulating androgen [4]. Local increased DHT synthesis caused by increased $5 \alpha$-reductase activity in the sebaceous glands may be associated with this phenomenon.

There are two subtypes of $5 \alpha$-reductase, steroid $5 \alpha$ reductase type 1 (SRD5A1) and steroid $5 \alpha$-reductase type 2

Address for correspondence: Dajin Zou, Department of Endocrinology, Changhai Hospital Affiliated to Second Military Medical University, 168 Chang'Hai Road, Shanghai 200433, China, phone: +86 21 31161391, e-mail: zwjd22@126.com, Yue Chen, Department of Endocrinology, Shanghai Baoshan Traditional Chinese Medicine-integrated Hospital, 181 You'Yi Road, 201900 Shanghai, China, phone: +86 2156601100 , e-mail: zwjd22@medmail.com.cn, moonchen2000@126.com Received: 22.12.2016, accepted: 19.05.2017. 
(SRD5A2), encoded by two distinct genes. SRD5A2 is located within the inner walls of open and closed comedones, and in endothelial cells from sections of inflammatory lesions from acne patients [5]. Acne patients obtain improvement with the treatment of finasterideSRD5A2 inhibitor by reducing $5 \alpha$-reductase activity and thus inhibiting the conversion of testosterone into DHT [6]. Therefore, we hypothesized that excessive local androgen production due to increased SRD5A2 activity in acne-prone skin plays a key role in the development of acne. Familial aggregation suggests the possibility of genetic susceptibility in such patients to develop acne $[7,8]$. Therefore, we hypothesized that genetic variation in the gene coding for SRD5A2 may be associated with acne pathogenesis.

The SRD5A2 gene is located on chromosome 2 at locus 2p23, containing 5 exons and 4 introns, encoding 254 amino acids, with a long 3' untranslated region. Several studies have reported that V89L (rs523349) polymorphism of the SRD5A2 gene was associated with the enzyme activity. Replacement of the amino acid valine with leucine downregulated the conversion rates of testosterone to DHT in the prostate, which reduced the risk of prostate cancer [9-11]. The (TA)n marker is located in the 3' untranslated region (3' UTR) of the SRD5A2 gene, and its functional consequence is thought to result in instability of mRNA transcripts with UA-rich 3' UTRs (transcribed from TA-rich regions of DNA), which may lead to dysregulated levels of reductase $[12,13]$. A study of 30 prostate cancer patients showed that almost $57 \%$ of the samples examined showed evidence of somatic mutations at the 3' UTR of the SRD5A2 locus [14].

\section{Aim}

In this study, genotyping of rs523349 and (TA)n of SRD5A2 was conducted in 49 acne patients with normal serum testosterone levels, who had significant improvements with SRD5A2 inhibitor-finasteride, and in 50 healthy age-matched Chinese controls without acne. Our focus is to investigate whether there is any association between V89L, TA dinucleotide repeat polymorphisms of the human SRD5A2 gene and the risk of developing acne in patients with a normal testosterone level, which has never been reported.

\section{Material and methods}

\section{Subjects}

A total of 99 individuals younger than 40 years old were recruited to participate in the study in Shanghai, China. They were from the Han ethnic group: 49 were patients presenting with acne and 50 were healthy control individuals with no acne history. The selection criteria for the acne patients for the study were: (1) the duration of symptoms was more than 6 months; (2) normal se- rum total testosterone (TT) and free testosterone (FT); (3) normal serum level of prolactin (PRL); (4) no evidence of polycystic ovarian syndrome, which is ruled out by regular menstrual cycles, normal ultrasound examination, and serum luteinizing hormone/follicle-stimulating hormone $(\mathrm{LH} / \mathrm{FSH})$ ratio < 1 ; (5) absence of chronic renal disease, diabetes mellitus, and hepatic disease; (6) after 3 months of treatment ( $5 \mathrm{mg}$ /day) with finasteride (a synthetic $5 \alpha$-reductase inhibitor from ProscarR; Merck, Sharp, Dohme Ltd, Hertfordshire, UK) in both men and women, skin lesions were improved significantly in both men and women. Female patients were informed that finasteride could increase the risk of birth defects in a male fetus and consequently pregnancy was contraindicated during the treatment and reliable contraception measures must be used such as the rhythm method and use of condoms, while any use of contraceptives with anti-androgen effects would be excluded. The exclusion criteria were the following: (1) occupational acne or druginduced acne-like rash; (2) pregnant or lactating women or 6 months of pregnancy requirements. Urine pregnancy tests were performed to exclude pregnant women; (3) subjects who had used any other drugs for treatment of acne in the last month, such as antibiotics, other antiandrogen drugs, glucocorticoids, and isotretinoin soft capsules.

All the patients and controls were enrolled with informed written consent. The study was approved by the Ethics Committee of Changhai Hospital and the procedures followed were in accordance with the Helsinki Declaration of 1975 as revised in 2000. All the patients signed informed consent for their participation in our study after reviewing the protocol of this experiment. Female patients were informed that finasteride could increase the risk of birth defects in a male fetus and consequently pregnancy was contraindicated during the treatment and the following 6 months, so reliable contraception must be used. We recorded the following information of the subjects: name, gender, age, height, weight, duration of disease, acne severity assessed by the Global Acne Grading System, acne genetic history, serum testosterone levels.

\section{Hormonal evaluation}

Blood samples were collected before the treatment with finasteride to evaluate estradiol (E), TT, FT, PRL, LH, $\mathrm{FSH}$, and insulin. All blood samples from female patients were obtained on the fifth day of the menstrual cycle.

\section{Efficacy evaluation of finasteride in the treatment of acne}

All patients were evaluated by Doshi's Global Acne Grading System (GAGS) [15]. We calculated the total score before and after the treatment, and evaluated the therapeutic effects according to changes in scores. The 
formula is efficacy index $=$ (pre-treatment score - posttreatment score) pre-treatment score $\times 100 \%$. An efficacy index more than $60 \%$ means significant effects.

\section{DNA extraction and genotype analysis}

DNA was extracted from whole blood samples using QIAamp DNA blood Kits (Qiagen, Valencia, CA). Polymerase chain reaction (PCR) was performed to determine V89L substitution. Primers were designed using the Primer-3 software as follows: a forward primer (5'-CAGCCGCTTGTCAACTCTCT-3'), a reverse primers (5'-ACGGTACTTCTGGGCCTCTT-3'), and amplification length was $133 \mathrm{bp}$. Amplifications were performed in a T-Gradient thermocycler (Biometra, Germany) as follows: (i) initial denaturation of $96^{\circ} \mathrm{C}$ for 5 min; 5 cycles of $96^{\circ} \mathrm{C}$ for $20 \mathrm{~s}, 68^{\circ} \mathrm{C}$ for $1 \mathrm{~min}$; (ii) 10 cycles of $96^{\circ} \mathrm{C}$ for $20 \mathrm{~s}, 64^{\circ} \mathrm{C}$ for $50 \mathrm{~s}, 72^{\circ} \mathrm{C}$ for $45 \mathrm{~s}$; (iii) 15 cycles of $96^{\circ} \mathrm{C}$ for $20 \mathrm{~s}, 61^{\circ} \mathrm{C}$ for $50 \mathrm{~s}, 72^{\circ} \mathrm{C}$ for $45 \mathrm{~s}$; (iv) an final extension at $72^{\circ} \mathrm{C}$ for $5 \mathrm{~min}$. Polymerase chain reaction reactions contained genomic DNA $0.5 \mu$, the primer (upstream and downstream primer; $20 \mu \mathrm{M}$ ) $0.5 \mu$ l, Premix Ex Taq (Code: D335A, TaKaRa Japan) $10 \mu \mathrm{l}$, and sterile distilled water $9 \mu \mathrm{l}$. Agarose gel electrophoresis was performed to confirm the purity of each reaction product. The PCR product was then sequenced by Bio Corporation.

Assay SRD5A2 gene (TA)n dinucleotide repeat polymorphism was determined with the real-time SYBR Green PCR-HRM (polymerase chain reaction - high-resolution melting) method. Primers were designed using Primer 3Plus according to the SRD5A2 sequence. The forward primer was 5'-GCTGATGAAAACTGTCAAGCTGCTGA-3', the reverse primer was 5'-GCCAGCTGGCAGAACGCCAGGAGAC-3', and amplification length was 116 bp. Amplification was performed in a Rotor-Gene 6000 (Corbett, Australia) as follows: initial denaturation of $95^{\circ} \mathrm{C}$ for $10 \mathrm{~min} ; 40$ cycles of $95^{\circ} \mathrm{C}$ for $10 \mathrm{~s}$ and then $60^{\circ} \mathrm{C}$ for $30 \mathrm{~s}$; melt $72^{\circ} \mathrm{C} \rightarrow 95^{\circ} \mathrm{C}, 1^{\circ} \mathrm{C}$ each step; high-resolution melting $75^{\circ} \mathrm{C} \rightarrow 84^{\circ} \mathrm{C} 0.1^{\circ} \mathrm{C}$ each step. Polymerase chain reaction reactions contained genomic DNA $0.5 \mu \mathrm{l}$, the primers (upstream and downstream primer, $20 \mu \mathrm{M}$ ), $0.5 \mu \mathrm{l}$, Realtime PCR Master Mix (Realtime PCR Master Mix, SYBR Green,
ABI, USA) $10 \mu \mathrm{l}$, sterile distilled water $9 \mu \mathrm{l}$. Those samples with different DNA melting curves were sequenced to identify their genotypes. Then the genotypes of the rest samples were identified by analysis of DNA melting curves during the polymerase chain reaction.

\section{Statistical analysis}

We calculated the allelic percentages for SRD5A2 polymorphisms and used the $\chi^{2}$ test to calculate the significance of the differences between cases and controls. The $\chi^{2}$ values were calculated with SPSS 17.0 software. Two sided $p$-values of less than 0.05 were considered significant. Different alleles at V89L and the (TA)n polymorphic sites were not investigated for linkage disequilibrium in the cases and the controls, because the sample size was too small.

\section{Results}

There were no significant differences between acne cases and controls in age, gender or BMI (Table 1).

The V89L site was highly polymorphic. However, there was no significant difference between acne cases and controls in the frequency distribution of various alleles at this site (Table 2). For genotype analyses, Val/Val genotype was considered to be a high activity genotype, while $\mathrm{Val} / \mathrm{Leu}$ and Leu/Leu were considered to be low activity genotypes. We did not find any differences between the acne patient group and the control group in the distribution of high and low activity genotypes (Table 2).

At the (TA)n repeat site, five alleles of the (TA)n repeat - (TA)0, (TA)3, (TA)6, (TA)9 and (TA)12 - were detected in this study, labeled as alleles A, B, C, D and E. Eleven genotypes were present in the participants. Table 3 shows the distribution of the genotypes. The TA repeat number in the acne patient group was from 3 to 12 (mean: 7.19, median: 6). In the control group the number of TA repeats was from 0 to 12 (mean: 5.94, median: 6). By using the Cochran and Cox approximate $t$ test, a statistically significant difference of TA repeat numbers was found between the acne patient group and the control group (the Cochran and Cox

Table 1. Age, gender and BMI distribution among acne patients and healthy controls

\begin{tabular}{|c|c|c|c|c|c|c|c|}
\hline \multirow[t]{2}{*}{ Group } & \multicolumn{2}{|c|}{ Age [years] } & \multicolumn{3}{|c|}{ Gender } & \multicolumn{2}{|c|}{$\mathrm{BMI}\left[\mathrm{kg} / \mathrm{m}^{2}\right]$} \\
\hline & Mean \pm SD & $P$-value & Females & Males & $P$-value & Mean \pm SD & $P$-value \\
\hline Cases $(n=49)$ & $21.55 \pm 3.88$ & $>0.10$ & 38 & 11 & $>0.50$ & $20.50 \pm 1.82$ & $>0.5$ \\
\hline Controls $(n=50)$ & $22.18 \pm 3.37$ & & 36 & 14 & & $21.06 \pm 1.65$ & \\
\hline
\end{tabular}

Table 2. SRD5A2 rs523349 allele and genotype frequencies among acne patients and healthy controls

\begin{tabular}{|c|c|c|c|c|c|c|c|}
\hline \multirow[t]{2}{*}{ Group } & \multicolumn{3}{|c|}{ Allele frequencies } & \multicolumn{4}{|c|}{ Genotype frequencies } \\
\hline & V (\%) & L (\%) & $P$-value & VV (\%) & VL (\%) & LL (\%) & $P$-value \\
\hline Cases $(n=49)$ & 39.80 & 60.20 & $>0.5$ & 20.40 & 38.78 & 40.82 & $>0.5$ \\
\hline Controls $(n=50)$ & 43.00 & 57.00 & & 18.00 & 50.00 & 32.00 & \\
\hline
\end{tabular}


Table 3. Distribution of the different genotypes between control and case groups

\begin{tabular}{lcc}
\hline Genotypes & Controls & Cases \\
\hline$A A$ & 0 & 4 \\
\hline$A B$ & 0 & 2 \\
\hline$B B$ & 6 & 9 \\
\hline$B C$ & 0 & 7 \\
\hline$B D$ & 2 & 0 \\
\hline$C C$ & 17 & 7 \\
\hline$C D$ & 8 & 5 \\
\hline$C E$ & 0 & 1 \\
\hline$D D$ & 9 & 9 \\
\hline$D E$ & 3 & 4 \\
\hline$E E$ & 4 & 2 \\
\hline
\end{tabular}

approximate $t$ test, $p<0.05)$. With the TA repeat median number of 6 in the control group as the split point, we defined short TA repeat numbers $n<6$ as allele $S$ and long TA repeat numbers $n \geq 6$ as allele $L$. We found a significant difference between the acne patient group and the control group in the frequency distribution of various alleles and genotypes (Table 4). Individuals carrying the L allele of (TA) $\mathrm{n}$ had a higher risk of acne than those carrying the $\mathrm{S}$ allele $(\mathrm{OR}=3.52,95 \%$ confidence interval: 1.73-7.16).

\section{Discussion}

This study represents a preliminary investigation of the association between the variants of the androgen metabolic enzyme SRD5A2 gene and the risk of developing acne in the Chinese population. Subjects for the study were limited to patients with normal serum testosterone levels who had significant improvement after treatment with the SRD5A2 inhibitor finasteride. This suggests that the SRD5A2 enzyme activity may be an important mechanism in the pathogenesis of acne in these patients. Therefore, we were more likely to discover potential association(s) between polymorphism of the SRD5A2 gene and the risk of developing acne in the these patients, which would make our study practical in a reduced sample size.

SRD5A2 plays an important role in the conversion of testosterone to the more potent DHT, but it cannot function without NADPH as its cofactor [16]. Russell et al. [17] studied 22 nucleotide missense mutations of SRD5A2 gene and demonstrated that change of certain amino acids might affect activity of SRDSA2 through decreasing binding affinity of the enzyme for its substrate or $\mathrm{NADPH}$, or by affecting the optimum $\mathrm{pH}$ (which is $\mathrm{pH}$ 5.0-5.5). Given the important role of SRD5A2 in androgen metabolism, many investigators have hypothesized that increased $5 \alpha$-reductase activity might contribute to hyperandrogenism, and many studies have investigated the association of SRD5A2 gene variants and androgenrelated disorders. There have been reports on the V89L polymorphism and SRD5A2 activity and prostate cancer (an androgen-dependent neoplasm) risk, which showed that individuals carrying the $\mathrm{V}$ allele of the SRD5A2 gene had higher levels of plasma $5 \alpha$-androstan- $3 \beta, 17 \beta$-diol (a DHT metabolite) than those carrying the $L$ allele and that V89L substitution was associated with risk of prostate cancer $[10,18]$. An in vitro transfection experiment confirmed that the activity of $5 \alpha$-reductase with leucine at codon 89 was $47 \%$ lower than that with valine [19]. But the relationship between the V89L polymorphism and $5 \alpha$-reductase enzyme activity and prostate cancer risk remained controversial. Wang et al. conducted a recent meta-analysis of 25 reports (including 8615 prostate cancer cases and 9089 controls) on V89L polymorphism and prostate cancer risk, and revealed that males with VV or VL genotype were at increased risk for prostate cancer compared with those with LL genotype in the European population. But such a phenomenon was not found in populations from Asia and Africa [20].

Our study showed no significant difference between the acne group and the control group in either the frequency of $V$ and $L$ alleles of $V 89 L$ or the frequency of $V V$, $V L$ and LL genotypes of V89L, which implied that V89L might be a polymorphism with no effect on the binding affinity of the enzyme for the substrate or NADPH or the optimum $\mathrm{pH}$. However, there was a relatively small sample size in our study, so a study with a much larger sample size would be required to determine the significance of the V89L polymorphism for risk of acne.

SRD5A2 is primarily expressed in genital skin and the prostate. The polymorphisms of SRD5A2 have been studied in prostate diseases mostly. Reichardt et al. [21] reported 10 genotypes of fragments of (TA)n from $87 \mathrm{bp}$ to $131 \mathrm{bp}$ in prostate cancer patients of different races, including 37 low-risk Asian-Americans, 94 high-risk African-Americans, and 68 intermediate-risk non-Hispanic Whites. The longest fragments, from 121 bp to 131 bp, were detected only in African-Americans, who were the

Table 4. (TA)n allele and genotype distribution frequencies of SRD5A2 among acne patients and healthy controls

\begin{tabular}{|c|c|c|c|c|c|c|c|}
\hline \multirow[t]{2}{*}{ Group } & \multicolumn{3}{|c|}{ Allele frequencies } & \multicolumn{4}{|c|}{ Genotype frequencies } \\
\hline & $\mathrm{S}(\%)$ & L (\%) & $P$-value & SS (\%) & SL (\%) & LL (\%) & $P$-value \\
\hline Cases $(n=49)$ & 14.29 & 85.71 & $<0.005$ & 12.24 & 4.08 & 83.67 & $<0.025$ \\
\hline Controls $(n=50)$ & 37.00 & 63.00 & & 30.00 & 14.00 & 56.00 & \\
\hline
\end{tabular}


high-risk population. Rajender et al. [22] analyzed the (TA) $n$ repeat of the SRD5A2 gene in 87 histologically confirmed prostate cancer patients, 40 benign prostatic hyperplasia cases and 96 control samples from southern parts of India, and revealed that the (TA)9 allele might confer a certain prostate cancer risk in south Indian men. Thus, it has been proposed that longer TA repeat lengths may result in an elevation of enzyme activity, leading to an increased prostatic level of DHT. Our study showed five alleles of TA dinucleotide repeat polymorphism (TA)0, (TA)3, (TA)6, (TA)9, and (TA)12 - by PCR-HRM. The acne patients had longer TA repeats than controls. Our previous study demonstrated that young male acne patients with normal testosterone levels had higher DHT levels than controls, and with DHT levels significantly decreased after the treatment of finasteride, the disease was significantly improved [23]. That previous study has some relevance to this study. (TA)n polymorphism plays a role in the pathogenesis of acne in patients with elevated DHT levels.

This is the first genetic association analysis of the $5 \alpha$-reductase gene as a candidate gene for acne pathogenesis. This study showed that the V89L polymorphism in the SRD5A2 gene may have little effect on acne in the Chinese Han population, but the (TA)n repeat polymorphism in SRD5A2 may be associated with acne pathogenesis and longer (TA)n repeats may increase the risk of developing acne in the Chinese Han population. The (TA)n repeat polymorphism in SRD5A2 may be one of the genetic mechanisms involved in the pathogenesis of acne. Thus our study provides some mechanistic insight into the potential importance of SRD5A2 in acne pathogenesis. Simultaneous detection of TA repeat polymorphism and $5 \alpha$-reductase activity may provide a more accurate evaluation of the biological significance of this polymorphism. A study with a much larger sample size may be needed to determine such interactions in both normal people and acne patients.

\section{Acknowledgments}

Dajin Zou and Yue Chen contributed equally to this work.

This study was supported by postgraduate research funds of the Second Military Medical University of China.

We would like to thank the central laboratory of Changhai Hospital for carrying out the genetic testing in this study.

\section{Conflict of interest}

The authors declare no conflict of interest.

\section{References}

1. Zouboulis CC, Eady A, Philpott M, et al. What is the pathogenesis of acne? Exp Dermatol 2005; 14: 143-52.
2. Zouboutis CC. Is acne vulgaris a genuine inflammatory disease? Dermatology 2001; 203: 277-9.

3. Thiboutot D, Harris G, Lies V, et al. Activity of the type $15 \mathrm{a}$ reductase exhibits regional differences in isolated sebaceous glands and whole skin. J invest Dermatol 1995; 105: 209-14.

4. Deplewski D, Rosenfield RL. Role of hormones in pilosebaceous unit development. Endocr Rev 2000; 21: 363-92.

5. Thiboutot D, Bayne E, Thorne J, et al. Immunolocalization of 5alpha-reductase isozymes in acne lesions and normal skin. Arch Dermatol 2000; 136: 1125-9.

6. Kohler C, Tschumi K, Bodmer C, et al. Effect of finasteride $5 \mathrm{mg}$ (Proscar) on acne and alopecia in female patients with normal serum levels of free testosterone. Gynecol Endocrinol 2007; 23: 142-5.

7. Bataille V, Snieder H, MacGregor AJ, et al. The influence of genetics and environmental factors in the pathogenesis of acne: a twin study of acne in women. J Invest Dermatol 2002; 119: 1317-22.

8. Goulden V, McGeown CH, Cunliffe WJ. The familial risk of adult acne: a comparison between first-degree relatives of affected and unaffected individuals. Br J Dermatol 1999; 141: 297-300.

9. Nam RK, Toi A, Vesprini D, et al. V89L polymorphism of type-2,5-alpha reductase enzyme gene predicts prostate cancer presence and progression. Urology 2001; 57: 199-204.

10. Hsing AW, Chen C, Chokkalingam AP, et al. Polymorphic markers in the SRD5A2 gene and prostate cancer risk: a population-based case-control study. Cancer Epidemiol Biomarkers Prev 2001; 10: 1077-82.

11. Li Z, Habuchi T, Mitsumori K, et al. Association of V89L SRD5A2 polymorphism with prostate cancer development in a Japanese population. J Urol 2003; 169: 2378-81.

12. Makridakis NM, Ross RK, Pike MC, et al. Association of missense substitution in SRD5A2 gene with prostate cancer in African-American and Hispanic men in Los Angeles, USA. Lancet 1999; 354: 975-8.

13. Zubiaga AM, Belasco JG, Greenberg ME. The nonamer UUAUUUAUU is the key AU-rich sequence motif that mediates mRNA degradation. Mol Cell Biol 1995; 15: 2219-230.

14. Akalu A, DImajian DA, Highshaw RA, et al. Somatic mutations at the SRD5A2 locus encoding prostatic steroid 5alphareductase during prostate cancer progression. J Urol 1999; 161: 1355-8.

15. Doshi A, Zaheer A, Matthew J, et al. A comparison of current acne grading systems and proposal of a novel system. Internat J Dermatol 1997; 36: 416-8.

16. Mazen I, Gad Y, Hafez M, et al. Molecular analysis of 5alphareductase type 2 gene in eight unrelated Egyptian children with suspected 5 alpha-reductase deficiency: prevalence of the G34R mutation. Clin Endocrinol 2003; 58: 627-31.

17. Russell D, Wilson J. Steroid 5alpha-reductase: two genes/ two enzymes. Annu Rev Biochem 1994; 63: 25-61.

18. Makridakis N, Ross RK, Pike MC, et al. A prevalent missense substitution that modulates activity of prostatic steroid $5 \mathrm{a}$ reductase. Cancer Res 2000; 57: 1020-2.

19. Makridakis NM, di Salle E, Reichardt JK. Biochemical and pharmacogenetic dissection of human steroid 5alpa-reductase type II. Pharmacogenetics 2000; 10: 407-13.

20. Wang C, Tao W, Chen Q, et al. SRD5A2 V89L polymorphism and prostate cancer risk: a meta-analysis. Prostate 2010; 70: $170-8$.

21. Reichardt JKV, Makridakis N, Henderson BE, et al. Genetic variability of the human SRD5A2 gene: implications for prostate cancer risk. Cancer Res 1995; 55: 3973-5. 
22. Rajender S, Vijayalakshmi K, Pooja S, et al. Longer (TA)n repeat but not A49T and V89L polymorphisms in SRD5A2 gene may confer prostate cancer risk in South Indian men. J Androl 2009; 30: 703-10.

23. Xinye J, Wei $\mathrm{D}$, Xue H, et al. Changes of sex hormone levels in male acne patients with normal serum testosterone and effect of antiandrogen therapy. Acad I Sec Mil Med Univ 2009; 30: 1131-5. 